\title{
Transcriptional regulation of xenobiotic detoxification in Drosophila
}

\author{
Jyoti R. Misra, Michael A. Horner, Geanette Lam, and Carl S. Thummel ${ }^{\mathbf{1}}$ \\ Department of Human Genetics, University of Utah School of Medicine, Salt Lake City, Utah 84112, USA
}

Living organisms, from bacteria to humans, display a coordinated transcriptional response to xenobiotic exposure, inducing enzymes and transporters that facilitate detoxification. Several transcription factors have been identified in vertebrates that contribute to this regulatory response. In contrast, little is known about this pathway in insects. Here we show that the Drosophila Nrf2 (NF-E2-related factor 2) ortholog CncC (cap ' $n$ ' collar isoform-C) is a central regulator of xenobiotic detoxification responses. A binding site for $\mathrm{CncC}$ and its heterodimer partner Maf (muscle aponeurosis fibromatosis) is sufficient and necessary for robust transcriptional responses to three xenobiotic compounds: phenobarbital ( $\mathrm{PB})$, chlorpromazine, and caffeine. Genetic manipulations that alter the levels of CncC or its negative regulator, Keap1 (Kelch-like ECH-associated protein 1), lead to predictable changes in xenobiotic-inducible gene expression. Transcriptional profiling studies reveal that more than half of the genes regulated by $\mathrm{PB}$ are also controlled by $\mathrm{CncC}$. Consistent with these effects on detoxification gene expression, activation of the CncC/Keap1 pathway in Drosophila is sufficient to confer resistance to the lethal effects of the pesticide malathion. These studies establish a molecular mechanism for the regulation of xenobiotic detoxification in Drosophila and have implications for controlling insect populations and the spread of insect-borne human diseases.

[Keywords: xenobiotics; gene regulation; transcriptional control; Nrf2; insecticide resistance]

Supplemental material is available for this article.

Received June 22, 2011; revised version accepted August 5, 2011.

Toxic compounds in the environment pose a constant challenge to the survival of all living organisms. These toxins, referred to as xenobiotics, enter the body by physical contact, inhalation, or ingestion, and can originate from a wide range of sources, including pharmaceuticals, pesticides, plant toxins, and pollutants. Animals defend themselves against these compounds through an elaborate three-phase detoxification system, metabolizing xenobiotics into less harmful substances and facilitating their excretion (Xu et al. 2005). The phase I detoxification enzymes represent the most abundant class of xenobiotic-metabolizing enzymes. They consist of cytochrome P450 monooxygenases (P450s), which decrease the biological activity of a broad range of substrates (or, less often, increase their toxicity). The phase II enzymes act on the toxic by-products of the phase I response and include glutathione S-transferases (GSTs), UDP-glucuronosyltransferases (UGTs), and carboxylesterases. GSTs and UGTs add bulky side groups onto toxic compounds to increase their hydrophilicity, facilitating their excretion from the organism, while carboxylesterases catalyze the hydrolysis of ester-containing xenobiotics, leading to

${ }^{1}$ Corresponding author.

E-mail carl.thummel@genetics.utah.edu.

Article is online at http://www.genesdev.org/cgi/doi/10.1101/gad.17280911. their detoxification. The phase III system consists of ATPbinding cassette $(\mathrm{ABC})$ and other transmembrane transporters that actively export the conjugated toxins out of the cell.

Exposure of organisms to xenobiotics induces a widespread transcriptional response that up-regulates the expression of the detoxification machinery. Nuclear receptors play a central role in this pathway in mammals; in particular, the xenobiotic nuclear receptors pregnane $\mathrm{X}$ receptor (PXR, NR1I2) and constitutive androstane receptor (CAR, NR1I3) (Maglich et al. 2002). PXR and CAR regulate genes encoding all three classes of drug-metabolizing enzymes, including P450s and transporters. The basic helix-loop-helix (bHLH)-PAS domain transcription factors aryl hydrocarbon receptor (AHR) and its heterodimer partner, AHR nuclear translocator (ARNT), also regulate detoxification genes (Hankinson 1995; Rowlands and Gustafsson 1997). TCDD (2,3,7,8-tetrachlorodibenzop-dioxin) and polycyclic aromatic hydrocarbons act as ligands for AHR, and functional studies of AHR provide much of what we know about the toxic and carcinogenic effects of these compounds (Schecter et al. 2006). A number of other transcription factors have also been implicated in the regulation of detoxification gene expression, including the FXR, VDR, and HNF4 nuclear receptors (Xu et al. 2005; Pascussi et al. 2008), and the Nrf2 (NF-E2-related 
factor 2) CNC-bZIP transcription factor (Nguyen et al. 2009; Sykiotis and Bohmann 2010). These effects, however, appear to be more restricted than those conferred by PXR, CAR, and AHR/ARNT (Pascussi et al. 2008).

Remarkably, in contrast to the detailed studies in humans and mice, relatively little is known about the transcriptional regulation of xenobiotic responses in the fruit fly Drosophila. Rather, most studies of xenobiotic responses in insects have focused on adaptive responseshow strains of insects arise under selective pressure to become resistant to toxic compounds in their environment (Perry et al. 2011). For example, overexpression of a single P450 gene, Cyp6g1, is sufficient to confer DDT resistance in Drosophila (Daborn et al. 2002). This emphasis on adaptive responses to xenobiotics arises from the importance of insecticide resistance, which remains the main impediment for effective crop protection and the control of insect-borne human diseases such as malaria.

Like other animals, insects can regulate detoxification gene transcription in response to xenobiotic challenge. Several studies have addressed the mechanisms that underlie this regulation, mapping critical promoter elements that are required for P450 gene induction in response to pesticides or the well-studied xenobiotic phenobarbital (PB) (Brun et al. 1996; Maitra et al. 1996; Danielson et al. 1997; Dunkov et al. 1997; Dombrowski et al. 1998; McDonnell et al. 2004; Brown et al. 2005; Morra et al. 2010). No major trans-acting factors, however, have yet been identified that mediate this regulation. In an effort to address this issue, functional studies were undertaken to examine the single Drosophila ortholog of PXR and CAR, the DHR96 (NR1J1) nuclear receptor (King-Jones et al. 2006). Unexpectedly, however, only 10\% of the genes regulated by PB in wild-type flies are dependent on DHR96 for their proper transcriptional response to the drug. Moreover, these DHR96-regulated genes still display drug-induced transcription in mutant animals, albeit at a lower level than is seen in wild-type controls. These observations raise the important possibility that additional factors are involved in xenobioticresponsive gene regulation.

Here we identify the Nrf2 ortholog cap ' $n$ ' collar isoform-C ( $\mathrm{CncC})$ as a central regulator of xenobiotic responses in Drosophila. Nrf2 plays an important role in regulating cellular defenses against oxidative and electrophilic stress (Nguyen et al. 2009; Sykiotis and Bohmann 2010). In the absence of stress, Nrf2 is retained in the cytoplasm by the actin-binding protein Keap1 (Kelch-like $\mathrm{ECH}$-associated protein 1), which also functions as an E3 ubiquitin ligase to promote Nrf2 degradation by the $26 \mathrm{~S}$ proteasome. Activation of the pathway disrupts the Nrf2-Keap1 interaction, allowing Nrf2 to translocate to the nucleus, where it can heterodimerize with the small Maf (muscle aponeurosis fibromatosis) proteins and bind to antioxidant response elements (AREs) in the genome. Nrf2, Maf, and Keap1 are all conserved in Drosophila and appear to exert the same regulatory interactions as defined in vertebrates (Sykiotis and Bohmann 2008, 2010).
Using a combination of promoter mapping in transgenic animals, bioinformatics, and genetics, we show that the CncC/Keapl pathway is a central regulator of xenobiotic responses in Drosophila. Transcriptional profiling studies reveal that $\mathrm{CncC}$ regulation can account for $70 \%$ of the genes that are induced in response to $\mathrm{PB}$. Consistent with this, constitutive activation of the Nrf2/ Keap1 pathway confers resistance to the lethal effects of the insecticide malathion. These studies establish Nrf2 as a key regulator of xenobiotic responses in insects and provide a foundation for using Drosophila as a model system to characterize this pathway. These studies also have implications for understanding the mechanisms of acquired pesticide resistance and its impact on effective crop protection and the control of insect-borne human diseases.

\section{Results}

Xenobiotics induce a coordinated transcriptional response in Drosophila

Although PB has been shown to direct changes in gene expression in Drosophila, none of these studies have addressed the timing or coordination of this transcriptional response (King-Jones et al. 2006; Sun et al. 2006; Willoughby et al. 2006). As a first step toward defining the molecular mechanisms of xenobiotic-regulated transcription in Drosophila, nine genes were selected to characterize their transcriptional regulation by $\mathrm{PB}$. Four of these genes encode P450s: Cyp6a2, Cyp6a8, Cyp6a21, and Cyp12d1. Cyp12d1 overexpression provides resistance to DDT and dicyclanil (Daborn et al. 2007). We also examined two GST genes (GstD2 and GstD7), the UGT encoded by CG5724, and two genes that do not encode phase I/phase II detoxification enzymes (Jheh1 and CG6188). Theh1 encodes an epoxide hydrolase, representing a class of enzymes that can detoxify epoxides in mammals, while CG6188 encodes a glycine N-methyltransferase, which can bind to carcinogenic polycyclic aromatic hydrocarbons and contribute to P450 induction (Bhat and Bresnick 1997).

The dose response profile of $\mathrm{PB}$-induced transcription was examined by feeding wild-type flies with different $\mathrm{PB}$ concentrations, from $0.003 \%$ to $1.0 \%$. RNA samples were then isolated and analyzed by Northern blot hybridization to detect expression of the nine detoxification genes (Fig. 1A). Very similar dose responses are observed under these conditions, with most genes showing detectable induction at $0.01 \% \mathrm{~PB}$ and efficient induction by $0.03 \%$. Wild-type flies treated with $0.1 \%$ PB or lower display no detectable changes in behavior, while reduced activity and incoordination are observed at higher $\mathrm{PB}$ concentrations (King-Jones et al. 2006). Thus, the transcriptional responses of these genes are more sensitive than the behavioral response, consistent with their proposed defensive function.

The time course of PB-induced transcription was also examined by feeding wild-type flies with $0.3 \%$ PB for $0.5-$ $6 \mathrm{~h}$, after which RNA samples were analyzed by Northern 


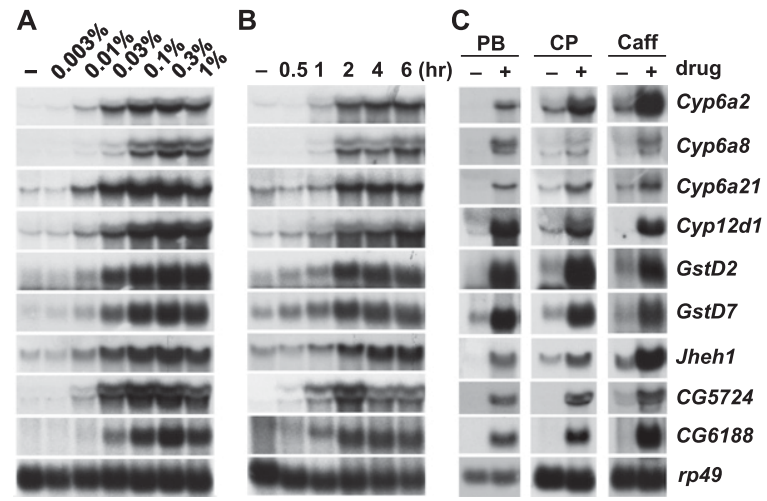

Figure 1. Xenobiotics induce a coordinated transcriptional response in Drosophila. (A) Wild-type (CanS) flies were treated with either no $\mathrm{PB}(-)$ or different concentrations of $\mathrm{PB}$, as indicated, for $6 \mathrm{~h}$, after which RNA was extracted and analyzed by Northern blot hybridization to detect the transcription of PBinducible genes, as shown. $(B)$ Wild-type flies were treated with either no $\mathrm{PB}(-)$ or $0.3 \% \mathrm{~PB}$ for the indicated time periods, after which RNA was extracted and analyzed by Northern blot hybridization to detect the transcription of PB-inducible genes. (C) Adult CanS flies were treated with no PB (-) or either $0.3 \%$ $\mathrm{PB}, 0.3 \%$ chlorpromazine $(\mathrm{CP})$, or $1.5 \mathrm{mg} / \mathrm{mL}$ caffeine (Caff), as indicated $(+)$, for $6 \mathrm{~h}$, after which RNA was extracted and analyzed by Northern blot hybridization to detect the transcription of PB-inducible genes. Hybridization to detect $r p 49$ mRNA was used as a control for loading and transfer in all panels.

blot hybridization (Fig. 1B). An initial increase in transcript levels is evident by $1 \mathrm{~h}$ of $\mathrm{PB}$ treatment, with a maximal response by $2 \mathrm{~h}$. Cyp6a21, Cyp12d1, GstD2, GstD7, and Theh1 show a basal level of expression, upon which transcript levels increase significantly following PB treatment (Fig. 1B). Taken together with the dose response study, these results indicate that $\mathrm{PB}$ directs a rapid and coordinated transcriptional program, consistent with a response to one or a few key transcription factors.

We also tested two different compounds, chlorpromazine and caffeine, for their effects on detoxification gene expression. Like $\mathrm{PB}$, chlorpromazine is a sedative (marketed as Thorazine and used as a xenobiotic in vertebrate studies) (e.g., Wei et al. 2002). PB and chlorpromazine, however, have different chemical structures and different modes of action. Whereas PB acts by increasing the chloride current from the GABA receptor, enhancing the effects of this inhibitory neurotransmitter, chlorpromazine interferes with dopaminergic pathways in the brain (Morrison and Murray 2005). In contrast, caffeine is a xanthine alkaloid compound that acts as an antagonist of adenosine receptors in the brain, resulting in increased dopamine activity (Cauli and Morelli 2005). Adult flies were treated with $\mathrm{PB}$, chlorpromazine, or caffeine, after which RNA was extracted and analyzed by Northern blot hybridization (Fig. 1C). The resulting pattern of induction by chlorpromazine and caffeine is very similar to that of $\mathrm{PB}$, suggesting that the transcriptional changes induced by $\mathrm{PB}$ are not specific to this compound, but rather represent a general xenobiotic detoxification response.
The bHLH-PAS transcription factor Methoprene tolerant (Met) does not contribute to PB-induced transcriptional responses

The apparent role of AHR and ARNT bHLH-PAS domain transcription factors in controlling mammalian xenobiotic detoxification prompted us to examine the possibility that this function is conserved through evolution. The Drosophila Spineless (Ss) protein provides the best match to the bHLH-PAS domain of AHR, with $45 \%$ identity across this region. Genetic studies of Ss, however, indicate that it plays an essential developmental role in leg and antennal specification, and suggest that it has no function in xenobiotic pathways (Duncan et al. 1998; McMillan and Bradfield 2007). A more likely candidate for regulating xenobiotic responses is the Drosophila bHLH-PAS gene Met. Met was identified in an openended genetic screen for mutations that allow flies to survive a lethal dose of the $\mathrm{JH}$ analog methoprene (widely used as a commercial pesticide) (Wilson and Fabian 1986). The subsequent determination that Met is an AHR homolog raised the possibility that it could function in a xenobiotic response pathway (Ashok et al. 1998). We thus examined the expression of several PB-inducible genes in animals carrying the $M e t^{1}$-null mutation (Fig. 2A). Cyp6a21, Theh1, GstD7, and GstD2 show a pattern of PB

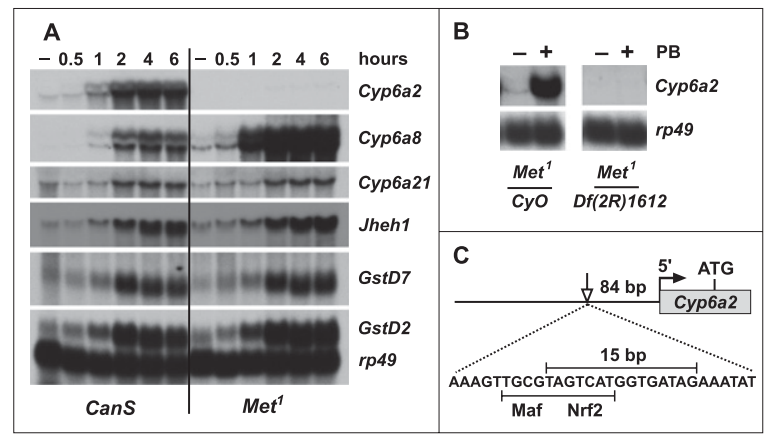

Figure 2. A 15-bp element in the Cyp6a2 promoter is necessary for transcription. (A) Adult CanS flies or $\mathrm{Met}^{1}$-null mutants were treated with either no $\mathrm{PB}(-)$ or $\mathrm{PB}$ for the indicated time periods, after which RNA was extracted and analyzed by Northern blot hybridization to detect the transcription of $\mathrm{PB}$ inducible genes. Although Cyp6a2 does not appear to be expressed in $\mathrm{Met}^{1}$ mutants, full-length mRNA is detectable upon longer exposure. $(B)$ The $M e t^{1}$ flies were crossed to a stock carrying a second chromosome $\mathrm{CyO}$ balancer that carries a wildtype Cyp6a2 locus (left panel), or a stock carrying the $D f(2 R) 1612$ deficiency that removes the Cyp6a2 locus (right panel), and the progeny from these crosses were treated with either no $\mathrm{PB}(-)$ or $\mathrm{PB}(+)$ for $4 \mathrm{~h}$, after which RNA was extracted and analyzed by Northern blot hybridization to detect Cyp6a2 mRNA. In all panels, hybridization to detect $r p 49$ mRNA was used as a control for loading and transfer. $(C)$ A schematic representation of the Cyp6a2 5' region is shown, with the transcribed sequences and ATG codon marked. Shown below is the sequence of a portion of the Cyp6a2 5' flanking region located 84 bp upstream of the start site of transcription (5' arrow), which was determined by $5^{\prime}$ RACE. The 15-bp sequence that is deleted in the $\mathrm{Met}^{1}$ mutants and the canonical Nrf2/Maf-binding site are marked by bracketed lines above and below the sequence, respectively. 
induction in $\mathrm{Met}^{1}$ mutants that is essentially indistinguishable from that seen in wild-type controls (Fig. 2A). Cyp6a8 appears to be induced to a higher level in $\mathrm{Met}^{1}$ mutants, although its timing is unchanged. In contrast, Cyp6a2 is expressed at very low levels in $\mathrm{Met}^{1}$ mutants treated with PB. A similarly low level of expression is seen in response to caffeine and chlorpromazine (Supplemental Fig. S1A). This gene is, however, induced normally in the null Met ${ }^{D 29}$ mutant as well as $\mathrm{Met}^{\mathrm{NG}}$ and $\mathrm{Met}^{3}$ mutants, indicating that this reduced expression is specific to the $\mathrm{Met}^{1}$ genetic background (Supplemental Fig. S2). Taken together, these results suggest that Met is not necessary for the transcriptional response to $\mathrm{PB}$.

\section{A 15-base-pair (bp) sequence in the Cyp6a2 promoter is necessary for expression}

The failure of $\mathrm{Met}^{1}$ mutants to express Cyp6a2 could be due to one or more trans-acting loci in the $\mathrm{Met}^{1}$ genetic background or a mutation linked to the Cyp6a2 locus. To distinguish between these possibilities, Met $^{1}$ flies were crossed with a stock that carries a second chromosome deficiency that removes the Cyp6a2 region, and the offspring were analyzed for Сyp6a2 transcription after treatment with PB (Fig. 2B). Cyp6a2 induction by PB appears normal when the second chromosome of $\mathrm{Met}^{1}$ is carried over a second chromosome balancer that includes a wild-type Cyp6a2 locus (Fig. 2B, left panel), but not when the $\mathrm{Met}^{1}$ second chromosome is carried in combination with a deficiency for Cyp6a2 (Fig. 2B, right panel), indicating that the loss of Cyp6a2 transcription in $\mathrm{Met}^{1}$ mutants is linked to the Cyp6a2 locus. Given that low levels of full-length Cyp6a2 mRNA can be detected in $M e t^{1}$ mutants, we reasoned that its reduced expression might be due to one or more mutations in its cis-regulatory sequences. To test this possibility, we sequenced $2 \mathrm{~kb}$ of $\mathrm{Met}^{1}$ genomic DNA upstream of Cyp6a2. This analysis revealed only one major difference from the wild-type sequence-a 15-bp deletion located 84 bp upstream of the Сур6а2 transcription start site (Fig. 2C). The possibility that this sequence is essential for Cyp6a2 promoter activity is consistent with independent promoter mapping studies of Cyp6a2 5' regulatory sequences. These studies showed that a Cyp $6 a 2$ promoter fragment extending from -1039 to $+23 \mathrm{bp}$ relative to the start site of transcription is sufficient to direct efficient PB induction of a lacZ reporter gene, comparable with the response of the endogenous Cyp6a2 gene (Supplemental Fig. S3). A similar response is seen with constructs that carry only $313 \mathrm{bp}$ or $158 \mathrm{bp}$ of $5^{\prime}$ flanking DNA, both of which include the 15-bp sequence that is deleted in $\mathrm{Met}^{1}$ mutants (Supplemental Fig. S3). These results indicate that key regulatory sequences required for $\mathrm{PB}$-inducible transcription map within 158 bp upstream of the Суp6a2 gene.

\section{The 15-bp element in the Cyp6a2 promoter contains a putative Nrf2/Maf-binding site that is necessary and sufficient for xenobiotic-induced transcription}

If the 15-bp region that is deleted in $\mathrm{Met}^{1}$ mutants has a regulatory function, then it could act as either a general enhancer required for promoter activity or, more importantly, as a binding site for a transcription factor that mediates xenobiotic detoxification. To test these possibilities, we established transgenic flies that carry a lac $Z$ reporter gene fused to either a wild-type 313-bp Cyp6a2 promoter fragment (WT-lacZ) or the same promoter fragment that carries the 15-bp deletion identified in the $\mathrm{Met}^{1}$ mutants $(\Delta 15$-lacZ). These animals were exposed to xenobiotics, and Cyp6a2 and lacZ expression was examined by Northern blot hybridization (Fig. 3A). The wild-type promoter shows a normal response to the drug, comparable with that of the endogenous Cyp6a2 locus, while the promoter fragment lacking the 15-bp sequence shows no detectable transcriptional response to either $\mathrm{PB}$, chlorpromazine, or caffeine (Fig. 3A). This observation indicates that the lack of Cyp6a2 expression
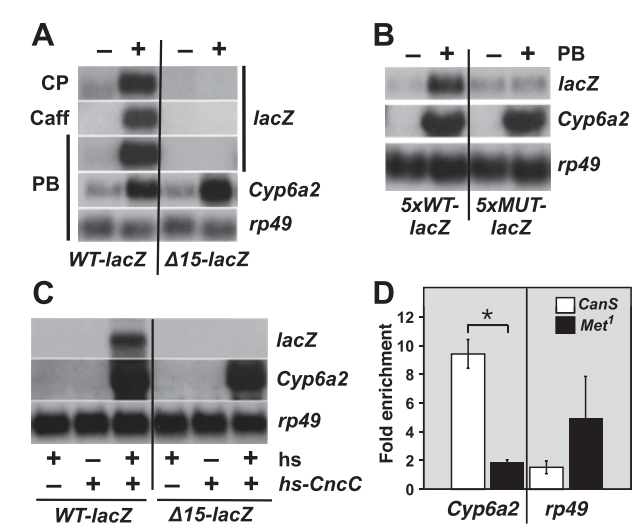

Figure 3. The Nrf2/Maf-binding site in the Cyp6a2 promoter is necessary and sufficient for its xenobiotic-induced transcription. (A) Transgenic animals carrying a lacZ reporter gene fused to either a wild-type 313-bp Cyp6a2 promoter fragment (WT-lacZ) or the same promoter fragment carrying the 15-bp deletion identified in the $\mathrm{Met}^{1}$ mutants $(\Delta 15$-lacZ) were fed with either no drug $(-)$ or $\mathrm{PB}$, chlorpromazine $(\mathrm{CP})$, or caffeine (Caff) for $6 \mathrm{~h}$ $(+)$, after which RNA was extracted and analyzed by Northern blot hybridization to detect $1 a c Z$ and Cyp6a2 transcription. (B) Transgenic animals carrying a lac $Z$ reporter gene fused to either five tandem copies of a 25-bp sequence that encompasses the Nrf2/Maf-binding site in the Cyp6a2 promoter (5XWT-1acZ) or a mutant version of this promoter fragment lacking the Nrf2/ Maf-binding site (5XMUT-lacZ) were fed with either no PB (-) or $\mathrm{PB}(+)$ for $6 \mathrm{~h}$, after which RNA was extracted and analyzed by Northern blot hybridization to detect Cyp6a2 and lacZ transcription. $(C)$ RNA was extracted from animals carrying either the WT-lacZ or the $\Delta 15$-lacZ transgenes, following either no heat treatment $(-\mathrm{hs})$ or heat treatment $(+\mathrm{hs})$, in the absence or presence of the hsp70-CncC transgene (hs-CncC). This RNA was analyzed by Northern blot hybridization to detect lac $Z$ and Cyp6a2 transcription. In all panels, hybridization to detect $r p 49$ mRNA was used as a control for loading and transfer. $(D)$ Cantons (CanS) control and $\mathrm{Met}^{1}$ mutant flies were fed with PB for $6 \mathrm{~h}$, after which they were homogenized and subjected to formaldehyde cross-linking, followed by sonication and ChIP. The presence of Cyp6a2 or rp49 (as a negative control) sequences was assayed by quantitative PCR (qPCR). Results from a representative experiment are shown. The $Y$-axis depicts the fold enrichment over a mock immunoprecipitation control that lacks Cnc antibody. $\left(^{\star}\right) P<0.015$. 
in $\mathrm{Met}^{1}$ mutants can be accounted for by the 15-bp deletion in the Cyp6a2 promoter.

MatInspector software was used to scan the region that encompasses the 15-bp sequence for potential transcription factor-binding sites (using a matrix similarity of $0.90)$. Interestingly, a single match was found, corresponding to the canonical binding site for the Nrf2/Maf heterodimer (Fig. 2C). The ability of this transcription factor to respond to oxidative and electrophilic stress, and its known role in regulating class II xenobiotic detoxification genes, raised the possibility that it might contribute to the drug inducibility of the Cyp6a2 promoter $(\mathrm{Hu}$ et al. 2006; Nguyen et al. 2009; Sykiotis and Bohmann 2010). To test this possibility, transgenic lines were established that carry a lac $Z$ reporter gene fused to either five tandem copies of a 25 -bp sequence that encompasses the 15-bp region, or a mutant version of this promoter fragment that carries point mutations disrupting the Nrf2/Maf-binding site (Veraksa et al. 2000). Northern blot analysis revealed efficient PB-inducible lacZ transcription in animals carrying the wild-type 25-bp sequence, but no detectable response in animals carrying the mutated sequence (Fig. 3B). This sequence is also sufficient to support highly inducible transcription in response to chlorpromazine and caffeine (Supplemental Fig. S1B). Taken together, these results suggest that the Nrf2/Maf-binding site in the Cyp6a2 promoter is both necessary and sufficient to mediate xenobiotic-inducible transcription.

The CncC/Keap1 pathway is necessary and sufficient for xenobiotic-induced transcription

The Nrf2 ortholog in Drosophila is encoded by the $c n c C$ isoform of the cnc locus (cnc-RC mRNA isoform on FlyBase) and can be inhibited through interactions with the fly Keap1 (Sykiotis and Bohmann 2008). Cnc proteins heterodimerize with Drosophila Maf-S in much the same manner as their mammalian counterparts, binding to a canonical Nrf2/Maf-binding site (Veraksa et al. 2000). Chromatin immunoprecipitation (ChIP) experiments using an antibody directed against the DNA-binding domain of Cnc revealed that this protein is bound to the Cyp6a2 promoter in wild-type flies but not to the same region in $\mathrm{Met}^{1}$ mutants, confirming that this sequence is a direct target for Cnc regulation (Fig. 3D).

If the CncC/Keap1 pathway is central to the drugregulated detoxification response, then overexpression of $\mathrm{CncC}$ should be sufficient to induce detoxification gene expression in the absence of drug, as should a loss of Keapl function (which would result in $\mathrm{CncC}$ stabilization). Conversely, a loss of $\mathrm{CncC}$ function should prevent the normal transcriptional response to xenobiotics, as would overexpression of Keapl (which enhances CncC degradation). These predictions were tested using the GAL4/UAS system to either overexpress or disrupt CncC or Keap1 function. Transgenic flies were established that carry the ubiquitous tubulin-GAL4 driver in combination with either UAS-CncC-RNAi, UAS-Keap1-RNAi, or UAS-Keap1. The Tub-Gal80ts construct was included in these lines to control the timing of $\mathrm{CncC}$ or Keap1 RNAi or Keap1 overexpression, as these conditions are normally lethal (Sykiotis and Bohmann 2008). A hsp70$\mathrm{CncC}$ transgene was used for heat-inducible $\mathrm{CncC}$ overexpression (McGinnis et al. 1998). Following temperature shifts to induce RNAi or overexpression, these animals were either left untreated or treated with $\mathrm{PB}$, after which RNA was extracted and examined by Northern blot hybridization to detect the expression of representative phase I and phase II detoxification genes. This study revealed that the $\mathrm{PB}$ induction of these genes is significantly attenuated by either CncC RNAi or Keap1 overexpression (Fig. 4A,C). While basal expression of hsp70$\mathrm{CncC}$ appears to direct weak induction of some of these genes in the absence of $\mathrm{PB}$, a dramatic response is seen upon heat induction (Fig. 4B). For Cyp6a2, this effect appears to be mediated by the Nrf2/Maf-binding site in its
A

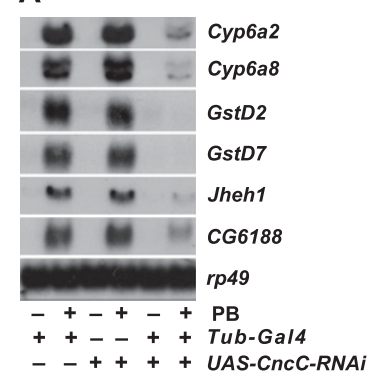

c

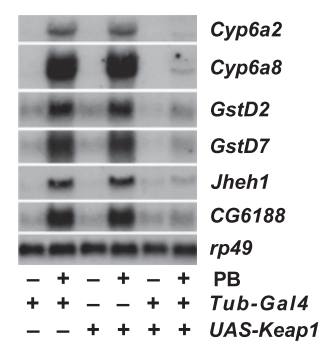

B

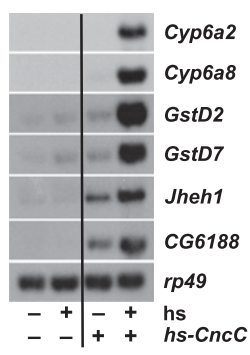

D

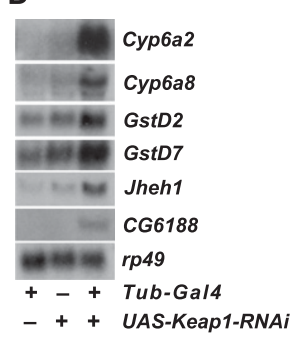

Figure 4. The CncC/Keapl pathway is necessary and sufficient for xenobiotic-induced transcription. (A) Flies carrying the TubGal80ts;Tub-GAL4 driver and/or UAS-CncC-RNAi transgene were shifted for $5 \mathrm{~d}$ to $29^{\circ} \mathrm{C}$, after which they were fed either no $\mathrm{PB}(-\mathrm{PB})$ or $\mathrm{PB}(+\mathrm{PB})$ for $6 \mathrm{~h}$. RNA extracted from these animals was analyzed by Northern blot hybridization to detect the transcription of PB-inducible genes, as shown. $(B)$ RNA was extracted from either control $w^{1118}$ animals (-hs-CncC) or transgenic animals carrying a heat-inducible copy of $\mathrm{CncC}(+h s-\mathrm{CncC})$ following either no heat treatment $(-\mathrm{hs})$ or heat treatment (+hs) and analyzed by Northern blot hybridization to detect the transcription of PB-inducible genes. $(C)$ Flies carrying the Tub-Gal80ts;Tub-GAL4 driver and/or UAS-Keap1 transgene were shifted for $4 \mathrm{~d}$ to $29^{\circ} \mathrm{C}$, after which they were fed either no $\mathrm{PB}$ $(-\mathrm{PB})$ or $\mathrm{PB}(+\mathrm{PB})$ for $4 \mathrm{~h}$. RNA extracted from these animals was analyzed by Northern blot hybridization to detect the transcription of PB-inducible genes. (D) Flies carrying the Tub-Gal80ts;TubGAL4 driver and/or UAS-Keap1-RNAi transgene were shifted for $4 \mathrm{~d}$ to $29^{\circ} \mathrm{C}$, after which RNA was extracted and analyzed by Northern blot hybridization to detect the transcription of PBinducible genes. Hybridization to detect $r p 49$ mRNA was used in all panels as a control for loading and transfer. 
promoter because the wild-type -313 -bp promoter-lacZ construct is induced efficiently by heat-induced $\mathrm{CncC}$ overexpression, while the construct lacking the 15-bp sequence shows no response (Fig. 3C). A similar upregulation of detoxification gene expression in the absence of drug treatment is seen in response to Keap1 RNAi (Fig. 4D). Taken together, these results confirm that the CncC/Keapl pathway is essential for the coordinate $\mathrm{PB}$-inducible expression of these six detoxification genes.

\section{CncC regulates the majority of $\mathrm{PB}$-inducible genes}

The central role of the $\mathrm{CncC} / \mathrm{Keap} 1$ pathway in PBinduced detoxification gene expression raises the interesting possibility that this pathway can account for most, if not all, xenobiotic-regulated transcription. To address this possibility, we conducted a microarray experiment to define the $\mathrm{CncC}$ transcriptional profile and compare this response to the set of genes regulated by $\mathrm{PB}$. Given that increased $\mathrm{CncC}$ protein levels are a key step in activation of the CncC/Keap1 pathway, we used heat-inducible CncC expression to define the CncC transcriptional program. RNA was isolated from control and transgenic hsp70-CncC flies following a brief heat treatment to induce CncC expression. In parallel, control flies were exposed to either sucrose alone or sucrose supplemented with PB. RNA was isolated from these two sets of animals, labeled, and hybridized to two-color Agilent Drosophila $44 \mathrm{~K}$ arrays. All experiments were conducted using four replicates to facilitate statistical analysis. This study revealed that 366 genes alter their expression twofold or greater in response to PB treatment, with 135 genes up-regulated and 231 genes down-regulated (Supplemental Table S1). As expected, this data set includes PB-inducible genes identified by other microarray studies (King-Jones et al. 2006; Sun et al. 2006; Willoughby et al. 2006). CncC overexpression results in 1406 transcripts that display a change in expression of at least twofold, with 712 genes up-regulated and 694 genes down-regulated (Supplemental Table S2). Importantly, most of the PB-regulated genes are also regulated by CncC (Fig. 5A), with $70 \%$ of the $\mathrm{PB}$ up-regulated genes also showing upregulation in response to $\mathrm{CncC}$ (Fig. 5B; Supplemental Table S3). As expected, these genes include all of those used in our Northern blot hybridization studies, validating the microarray results (Figs. 1, 4). Taken together, our results indicate that most of the transcriptional response to $\mathrm{PB}$ in Drosophila can be accounted for by activation of the CncC/Keapl pathway.

Representatives from all three classes of detoxification genes are regulated by both $\mathrm{PB}$ and $\mathrm{CncC}$ (Supplemental Tables S1,S2). This conclusion is supported by GOstat analysis of the CncC-regulated genes, which reveals oxidoreductase $(\mathrm{P} 450)$ activity, transferase activity, and transmembrane transporters in the top gene ontology (GO) categories of this data set (Fig. 5C). Significant overlaps are also seen with genes regulated by oxidative stress in Drosophila (Fig. 5C; Supplemental S4A,B; Girardot et al. 2004). This is consistent with an earlier microarray study

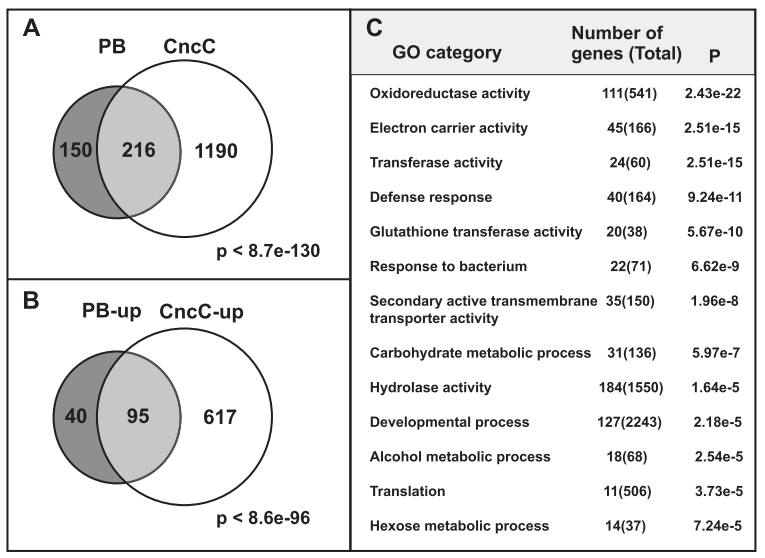

Figure 5. Most PB-regulated genes are also controlled by CncC. Venn diagrams are depicted that compare the genes that change their expression in wild-type flies treated with $\mathrm{PB}$ with genes that change their expression in response to ectopic CncC expression $(A)$, or the genes that are up-regulated in wild-type flies treated with $\mathrm{PB}$ (PB-up) with genes that are up-regulated in response to ectopic $\mathrm{CncC}$ expression (CncC-up) (B). The $P$-value for overlap of the gene sets is shown for each diagram. $(C)$ GOstat analysis of the genes that change expression in response to ectopic CncC expression. The top GO categories for each gene set are listed in order of significance along with the number of genes affected in that category, the total number of genes in that category (in parentheses), and the statistical significance of the match.

of the transcriptional response to $\mathrm{PB}$, as well as the known role of Nrf2/Maf transcription factors in mediating protective responses to oxidative stress (King-Jones et al. 2006; Nguyen et al. 2009; Sykiotis and Bohmann 2010). It also raises the possibility that oxidative stress is an intermediate in the xenobiotic response pathway. However, ubiquitous overexpression of either catalase, Sod1, or Sod2, each of which is known to reduce oxidative stress, has no effect on the transcriptional response to $\mathrm{PB}$ (Supplemental Fig. S5).

\section{Ectopic activation of the CncC/Keap1 pathway confers resistance against malathion}

Ectopic expression of several genes that are highly induced by $\mathrm{CncC}$ is sufficient to confer resistance to multiple pesticides (Daborn et al. 2001, 2007). We thus examined whether activation of the $\mathrm{CncC} / \mathrm{Keap} 1$ pathway can lead to increased insecticide resistance. Treatment with caffeine has no clear effect on Drosophila, and although negative geotaxis assays can be used to assess the sedative effects of drugs like PB and chlorpromazine, the results are variable and difficult to quantify. Accordingly, we examined the ability of insects to survive the lethal effects of the organophosphorus insecticide malathion. GAL4 drivers were used to direct Keap1 RNAi to several different tissues corresponding to the major sites of detoxification gene expression in Drosophila: the fat body, midgut, and Malpighian tubules (Chung et al. 2009; Perry et al. 2011). Although control animals die within $1 \mathrm{~d}$ following exposure to malathion, disruption of Keap1 
function in any of the major metabolic organs confers significant resistance to the drug (Fig. 6). This result indicates that activation of the $\mathrm{CncC} / \mathrm{Keap} 1$ signaling pathway is sufficient to confer resistance to malathion toxicity.

\section{Discussion}

Nrf2 is a major ancestral regulator of xenobiotic detoxification

In order to survive the continual threat of chemical toxins in their environment, animals have evolved complex and specific regulatory responses that include the coordinated transcriptional control of key detoxification genes. Insects are no exception, displaying a massive and rapid reprogramming of gene expression in response to xenobiotic challenge (Perry et al. 2011). The consequences of these detoxification responses in insects, however, have wide-ranging implications for human health and welfare. In particular, the emergence of insecticide-resistant strains has had a profound impact on both agricultural yields and the spread of insect-borne human diseases, with a disproportionate impact on developing countries. For example, more than 1 million people die each year from malaria, primarily in Africa. These devastating effects on human health have focused research efforts on defining the molecular mechanisms of xenobiotic detoxification in insects. As a result, many studies have been published that describe detailed promoter mapping of xenobiotic detoxification genes, identifying critical regulatory sequences needed for transcriptional responses to pesticides or PB (Brun et al. 1996; Maitra et al. 1996; Danielson et al.

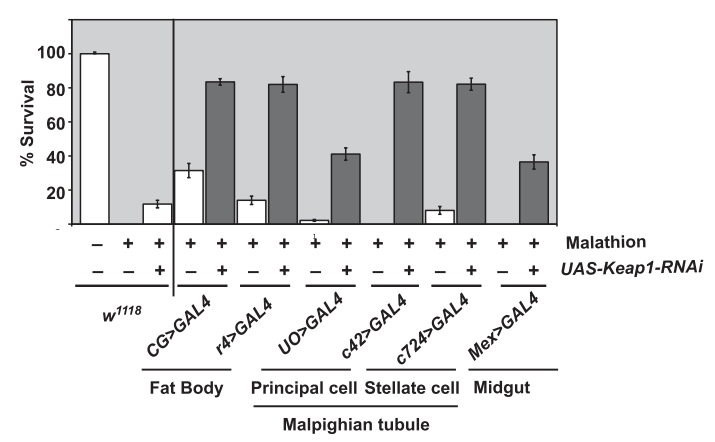

Figure 6. Activation of the $\mathrm{CncC} / \mathrm{Keap} 1$ pathway confers pesticide resistance. The GAL4/UAS system was used to activate the $\mathrm{CncC} / \mathrm{Keap} 1$ pathway in different tissues by inducing RNAi against Keap1, after which the animals were tested for resistance to the pesticide malathion. All experiments were done in the absence or presence of a UAS-Keap1-RNAi transgene in combination with different tissue-specific GAL4 drivers: $C G$ GAL4 and r4-GAL4 fat body drivers, UO-GAL4 and c42-GAL4 principal cell-specific Malpighian tubule drivers, C724-GAL4 stellate cell-specific Malpighian tubule driver, and Mex-GAL4 midgut driver. $w^{1118}$ animals were used as a control. The Y-axis represents the number of animals surviving after a 36-h exposure to $10 \mu \mathrm{M}$ malathion $(+)$. Ten replicates were used per genotype. White bars represent control animals, and gray bars represent animals in which the CncC/Keap1 pathway has been activated.
1997; Dunkov et al. 1997; Dombrowski et al. 1998; McDonnell et al. 2004; Brown et al. 2005; Morra et al. 2010). Although these studies have defined short segments of DNA that are required for promoter activity and implicated several transcription factors in mediating this response, no major trans-acting factors have yet been identified in insects. Here we show that the evolutionarily conserved Nrf2/Keap1 pathway plays a central role in insect detoxification responses.

Studies in mammals indicate that xenobiotic responses comprise a complex regulatory circuit that requires significant cooperation and cross-talk between multiple transcriptional regulators (Pascussi et al. 2008). In contrast, our studies suggest that xenobiotic responses have a more restricted regulatory input in insects and implicate the Nrf2/Keap1 pathway as a central ancestral regulator of xenobiotic detoxification. More than half of the genes regulated by $\mathrm{PB}$ in Drosophila are also controlled by $\mathrm{CncC}$, with a remarkable $70 \%$ of the genes upregulated by $\mathrm{PB}$ also up-regulated in response to $\mathrm{CncC}$ expression (Fig. 5). This increased correlation between the induced gene sets is consistent with the active nature of xenobiotic detoxification and the known role for increased enzymatic and transporter activity to facilitate toxin chemical modifications and export (Xu et al. 2005).

The widespread effects of Nrf2 on insect xenobiotic responses stands in sharp contrast to the Drosophila PXR homolog DHR96, where $\sim 10 \%$ of the genes regulated by $\mathrm{PB}$ are affected by a null mutation in this nuclear receptor (King-Jones et al. 2006). Similarly, our studies indicate no significant role for the Drosophila Met gene in xenobiotic detoxification (Fig. 2A). This is consistent with genetic studies of AHR homologs in mice, Ceanorhabditis elegans, and Drosophila (for a review, see McMillan and Bradfield 2007). Much like the role for the AHR homolog Ss in Drosophila antennal and leg development, AHR appears to have multiple developmental functions in mice (McMillan and Bradfield 2007). A current view is that the toxicity of TCDD may be due to its ectopic activation of AHR and the consequent interference with its normal developmental functions, rather than as a result of AHR acting as a xenobiotic receptor (McMillan and Bradfield 2007). More work is required to determine what role these factors play in normal xenobiotic detoxification. In addition, it is important to note that other regulatory mechanisms are likely to contribute to xenobiotic responses in insects. A number of tested compounds, including spinosad, diazinon, nitenpyram, lufenuron, and dicyclanil, have no effect on the expression of P450 and GST genes that are clearly responsive to PB and caffeine, while DDT has only a weak effect on a few of these genes (Willoughby et al. 2006). Further studies of these compounds should reveal whether and how they might modulate xenobiotic transcriptional responses.

\section{The CncC transcriptional program includes stress responses and metabolic control}

The xenobiotic transcriptional functions of mammalian Nrf2 are largely restricted to phase II genes (Itoh et al. 
1997; Hu et al. 2006). In contrast, our studies of fly CncC have demonstrated a widespread regulatory effect on detoxification pathways, with the top GO categories corresponding to phase I, phase II, and phase III responses (Fig. 5C). These include changes in the expression of 36 P450 genes, 17 GSTs, six UGTs, and 55 predicted transmembrane transporters (Supplemental Table S2). The three P450 genes that are sufficient to provide pesticide resistance-Cyp6g1, Cyp6g2, Cyp12d1-are highly upregulated in response to CncC (Daborn et al. 2002, 2007), as are all the genes selected for our original profiling of xenobiotic responses (Fig. 1) and genes examined for xenobiotic regulatory elements in Drosophila (Supplemental Table S2; Brun et al. 1996; Dombrowski et al. 1998; Morra et al. 2010). Although most of the CncCregulated predicted transporter genes are of unknown function, one- $d M R P$ - has been shown to act like its human homolog, as an ATP-dependent drug transporter (Szeri et al. 2009). Many cuticle genes are also up-regulated by $\mathrm{CncC}$ expression, providing possible protection against surface exposure to xenobiotic compounds (Supplemental Table S2). We also see a good correlation between our CncC-regulated gene set and the list of in vivo CncCbinding sites identified by modENCODE in Drosophila embryos using ChIP-chip (Supplemental Table S4; Negre et al. 2011). Of the 1406 CncC-regulated genes identified in our microarray study, 103 have a CncC-binding site within $2 \mathrm{~kb}$ of the gene region, with 67 of these sites lying within $500 \mathrm{bp}$. This confirms and extends our ChIP study of the Cyp6a2 promoter (Fig. 3D) and suggests that CncC plays a direct role in mediating xenobiotic detoxification responses.

As expected from its well-characterized role in mediating oxidative stress resistance, we see significant overlap between the set of genes regulated by $\mathrm{CncC}$ and genes that change their expression in response to treatment with either paraquat or hydrogen peroxide (Supplemental Fig. S4A,B; Girardot et al. 2004). This includes dramatic induction of Keap1, which is consistent with its role in negative feedback control of Nrf2 signaling. Interestingly, we also see down-regulation of many genes involved in innate immune protective responses (Supplemental Fig. S4C; Sackton and Clark 2009). This includes three cecropin genes, Mtk, Def, Drs, dro3, and other key effectors of the immune response. Although this is consistent with a recent study showing that antioxidants suppress innate immune responses, the significance of this repressive regulation remains unclear (Radyuk et al. 2010). It is interesting to note, however, that the oxidative, xenobiotic, and innate immune stress response pathways appear to be coordinated. Further studies are required to determine the molecular mechanisms and significance of this cross-regulation.

The set of CncC-regulated genes also includes many genes that play central roles in metabolism (Supplemental Table S5). Some of these functions are consistent with xenobiotic detoxification. Thus, for example, CncC upregulates $Z W$ and $P g d$, which encode glucose-6-phosphate dehydrogenase and phosphogluconate dehydrogenase in the pentose phosphate pathway. These enzymes are critical for NADPH production, which is essential for both P450 and GST function. It is also interesting to note that many genes involved in the breakdown of dietary carbohydrates and proteins are down-regulated along with the CG5932 gastric lipase and Npc1b cholesterol transporter, suggesting an overall suppression of midgut function. In addition, five of the eight $N p c 2$ genes change their expression in response to $\mathrm{CncC}$, with dramatic upregulation of $N p c 2 c$. The functions for these proteins remain unclear, although their similarity to mammalian Npc2 suggests a role in cellular sterol trafficking (Huang et al. 2007). Thus, the metabolic genes affected by the CncC/Keap1 pathway appear to represent both homeostatic responses required to maintain xenobiotic detoxification as well as protective functions to suppress dietary xenobiotic uptake and regulate trafficking.

\section{The CncC/Keap1 pathway provides a potential nodal point for insect population control}

A clear direction for future study will be to define the molecular mechanisms by which xenobiotic signals are transduced to result in activation of the CncC/Keap1 pathway. This is a significant challenge, as proteins that are capable of binding drugs like PB have evaded identification for many years. One possible model is that oxidative stress is an intermediate signal that connects xenobiotic exposure with activation of CncC. Our data, however, do not support this mechanism of action (Supplemental Fig. S5). Rather, a more likely avenue for gaining insights into this important level of control may be through mechanistic studies of acquired pesticide resistance.

Field-isolated and laboratory-selected strains of insecticide-resistant insects overexpress a number of detoxifying genes, demonstrating a correlation between their resistance and detoxification gene expression (FfrenchConstant et al. 2004; Li et al. 2007). Ectopic expression studies in Drosophila have supported this correlation. For example, overexpression of Cyp6g1 is sufficient to provide resistance to DDT, while Cyp12a4 overexpression provides resistance to Lufenuron (Daborn et al. 2002; Bogwitz et al. 2005). Consistent with this, activation of the CncC/Keapl pathway in key metabolic organs-the fat body, the midgut, and the Malpighian tubules-leads to malathion resistance (Fig. 6).

Taken together, these observations raise the important possibility that the $\mathrm{CncC} / \mathrm{Keap} 1$ pathway may provide a key nodal point for conferring insecticide resistance. This proposal is supported by the observation that some insecticide-resistant insect strains are resistant to oxidative stress and overexpress oxidative stress response genes, providing a functional link between these two pathways (Abdollahi et al. 2004; Vontas et al. 2005). Moreover, genetic mapping studies in DDT-resistant lines of Drosophila have identified one or more key trans-acting factors on the third chromosome that are required for the elevated expression of Cyp6a2 and Cyp6a8 (Maitra et al. 2000). This is consistent with another study that mapped a malathion resistance locus near the stripe locus at 90EF (Houpt et al. 1988). Interestingly, CncC and Keapl both 
map near this region on the third chromosome $94 \mathrm{E}$ and $89 \mathrm{E}$, respectively). It will be interesting to determine whether the CncC/Keapl pathway is activated in pesticide-resistant insect strains, whether this pathway plays a key role in conferring insecticide resistance, and whether chemical modulators of Nrf2 signaling can be used to control insect populations in the wild.

\section{Materials and methods}

Drosophila stocks

CanS, $w^{1118}, \mathrm{Met}^{1}, \mathrm{Met}^{\mathrm{N6}}, \mathrm{Met}^{\mathrm{D29}}, \mathrm{Met}^{3}, \mathrm{Df}(2 \mathrm{R}) \mathrm{ED} 1612, \mathrm{tub}-$ Gal80 ${ }^{\text {ts }}$, Tub-GAL4, CG-GAL4, r4-GAL4, Mex-GAL4, c724GAL4, UAS-cat, UAS-sod1, and UAS-sod2 were obtained from the Bloomington Drosophila Stock Center. UO-GAL4 and c42GAL4 were provided by S.A. Davies; UAS-Keap1, UAS-Keap1$R N A i$, and $U A S-C n c C-R N A i$ lines were provided by D. Bohmann; and $h s p 70-C n c C$ was provided by W. McGinnis. Flies were raised on standard cornmeal $/$ molasses/agar food at $20^{\circ} \mathrm{C}-25^{\circ} \mathrm{C}$. Stocks with tub-Gal80 ${ }^{\text {ts }}$ were reared at $18^{\circ} \mathrm{C}$. Recently eclosed adult flies were shifted for $3-6$ d to $29^{\circ} \mathrm{C}$ to allow GAL4 activation. Heat shock was carried out for $30 \mathrm{~min}$ at $37^{\circ} \mathrm{C}$ followed by $2 \mathrm{~h}$ of recovery at room temperature.

\section{Treatment with xenobiotic compounds and Northern} blot hybridizations

Newly eclosed flies ( $\leq 3$ d) that were raised on standard cornmeal/agar food were starved overnight under humid conditions and then exposed to xenobiotics. For xenobiotic exposure, 20 flies were placed in a glass scintillation vial with Whatman paper saturated with either $5 \%$ sucrose or $5 \%$ sucrose supplemented with $0.3 \% \mathrm{~PB}, 0.3 \%$ chlorpromazine, or $1.5 \mathrm{mg} / \mathrm{mL}$ caffeine (Sigma). After exposure, total RNA was isolated using Tripure (Roche). Equal amounts of total RNA were fractionated by formaldehyde agarose gel electrophoresis and analyzed by Northern blot hybridization, as described previously (King-Jones et al. 2006). Probes were generated by PCR, purified using Qiaquick gel extraction columns (Qiagen), and labeled with a Prime-It II kit (Stratagene). The PCR primers used to generate each probe are shown in the Supplemental Material.

\section{Microarray experiments}

To identify PB-regulated genes, RNA was isolated from mature adult $W^{1118}$ males fed with either sucrose alone or sucrose supplemented with $0.3 \% \mathrm{~PB}$, as described above. To identify CncC-regulated genes, RNA was isolated from either mature adult $w^{1118}$ males or $w$, hsp 70-CncC transformants that had been exposed for $30 \mathrm{~min}$ to $37^{\circ} \mathrm{C}$, followed by recovery for $2 \mathrm{~h}$ at $25^{\circ} \mathrm{C}$. All samples were prepared in four replicates to facilitate subsequent statistical analysis. Total RNA was extracted with TriPure (Roche) followed by purification with RNeasy columns (Qiagen). Probe labeling, hybridization to two-color Agilent Drosophila 44K arrays, and scanning were performed by the University of Utah Microarray Core Facility. The data were Lowess-normalized using $\mathrm{R}$, and the fold changes in gene expression and t-statistics were determined using GeneSifter (VizX Labs). $P$-values were calculated using the Benjamimi and Hochberg correction for false discovery rate. Comparison between microarray data sets was performed using Genevenn, and the $P$-value for significance of overlap between gene sets was calculated by hypergeometric probability. Microarray data from this study can be accessed at NCBI Gene Expression Omnibus (accession number: GSE30087).

\section{Malathion resistance assay}

Resistance to malathion was determined essentially as described, with the following exceptions (Houpt et al. 1988). Malathion (Chem Services) was dissolved in 2-propanol and added to a final concentration of $10 \mu \mathrm{M}$ in a solution of $1 \%$ agar and $5 \%$ sucrose. Twenty adult females ( 1 wk old) were transferred to vials containing $10 \mathrm{~mL}$ of this medium and maintained at $25^{\circ} \mathrm{C}$. The number of individuals surviving after $36 \mathrm{~h}$ was counted.

\section{Acknowledgments}

We thank the Bloomington Stock Center, S.A. Davies, D. Bohmann, and W. McGinnis for providing stocks; W. McGinnis for providing Cnc antibodies; and FlyBase for critical information that made these studies possible. We also thank M. Sieber and J. Tennessen for critical comments on the manuscript. This research was supported by the National Institute of General Medical Sciences (R01GM079197).

\section{References}

Abdollahi M, Ranjbar A, Shadnia S, Nikfar S, Rezaiee A. 2004. Pesticides and oxidative stress: a review. Med Sci Monit 10: RA141-RA147.

Ashok M, Turner C, Wilson TG. 1998. Insect juvenile hormone resistance gene homology with the bHLH-PAS family of transcriptional regulators. Proc Nat1 Acad Sci 95: 2761-2766.

Bhat R, Bresnick E. 1997. Glycine N-methyltransferase is an example of functional diversity. Role as a polycyclic aromatic hydrocarbon-binding receptor. I Biol Chem 272: 21221-21226.

Bogwitz MR, Chung H, Magoc L, Rigby S, Wong W, O'Keefe M, McKenzie JA, Batterham P, Daborn PJ. 2005. Cyp12a4 confers lufenuron resistance in a natural population of Drosophila melanogaster. Proc Natl Acad Sci 102: 1280712812.

Brown RP, McDonnell CM, Berenbaum MR, Schuler MA. 2005. Regulation of an insect cytochrome P450 monooxygenase gene (CYP6B1) by aryl hydrocarbon and xanthotoxin response cascades. Gene 358: 39-52.

Brun A, Cuany A, Le Mouel T, Berge J, Amichot M. 1996. Inducibility of the Drosophila melanogaster cytochrome P450 gene, CYP6A2, by phenobarbital in insecticide susceptible or resistant strains. Insect Biochem Mol Biol 26: 697703.

Cauli O, Morelli M. 2005. Caffeine and the dopaminergic system. Behav Pharmacol 16: 63-77.

Chung H, Sztal T, Pasricha S, Sridhar M, Batterham P, Daborn PJ. 2009. Characterization of Drosophila melanogaster cytochrome P450 genes. Proc Natl Acad Sci 106: 5731-5736.

Daborn P, Boundy S, Yen J, Pittendrigh B, ffrench-Constant R. 2001. DDT resistance in Drosophila correlates with Cyp6g1 over-expression and confers cross-resistance to the neonicotinoid imidacloprid. Mol Genet Genomics 266: 556-563.

Daborn PJ, Yen JL, Bogwitz MR, Le Goff G, Feil E, Jeffers S, Tijet N, Perry T, Heckel D, Batterham P, et al. 2002. A single P450 allele associated with insecticide resistance in Drosophila. Science 297: 2253-2256.

Daborn PJ, Lumb C, Boey A, Wong W, Ffrench-Constant RH, Batterham P. 2007. Evaluating the insecticide resistance potential of eight Drosophila melanogaster cytochrome P450 genes by transgenic over-expression. Insect Biochem Mol Biol 37: 512-519.

Danielson PB, MacIntyre RJ, Fogleman JC. 1997. Molecular cloning of a family of xenobiotic-inducible drosophilid cyto- 
chrome P450s: evidence for involvement in host-plant allelochemical resistance. Proc Natl Acad Sci 94: 1079710802 .

Dombrowski SM, Krishnan R, Witte M, Maitra S, Diesing C, Waters LC, Ganguly R. 1998. Constitutive and barbitalinduced expression of the Cyp6a2 allele of a high producer strain of CYP6A2 in the genetic background of a low producer strain. Gene 221: 69-77.

Duncan DM, Burgess EA, Duncan I. 1998. Control of distal antennal identity and tarsal development in Drosophila by spineless-aristapedia, a homolog of the mammalian dioxin receptor. Genes Dev 12: 1290-1303.

Dunkov BC, Guzov VM, Mocelin G, Shotkoski F, Brun A, Amichot M, Ffrench-Constant RH, Feyereisen R. 1997. The Drosophila cytochrome P450 gene Cyp6a2: structure, localization, heterologous expression, and induction by phenobarbital. DNA Cell Biol 16: 1345-1356.

Ffrench-Constant RH, Daborn PJ, Le Goff G. 2004. The genetics and genomics of insecticide resistance. Trends Genet 20: 163-170.

Girardot F, Monnier V, Tricoire H. 2004. Genome wide analysis of common and specific stress responses in adult Drosophila melanogaster. BMC Genomics 5: 74. doi: 10.1186/1471-21645-74.

Hankinson O. 1995. The aryl hydrocarbon receptor complex. Annu Rev Pharmacol Toxicol 35: 307-340.

Houpt DR, Pursey JC, Morton RA. 1988. Genes controlling malathion resistance in a laboratory-selected population of Drosophila melanogaster. Genome / NRC Canada 30: 844853.

Hu R, Xu C, Shen G, Jain MR, Khor TO, Gopalkirshnan A, Lin W, Reddy B, Chang JY, Kong AN. 2006. Identification of Nrf2-regulated genes induced by chemopreventive isothiocyanate PEITC by oligonucleotide microarray. Life Sci 79: $1944-1955$.

Huang X, Warren JT, Buchanan J, Gilbert LI, Scott MP. 2007. DrosophilaNiemann-Pick type C-2 genes control sterol homeostasis and steroid biosynthesis: a model of human neurodegenerative disease. Development 134: 3733-3742.

Itoh K, Chiba T, Takahashi S, Ishii T, Igarashi K, Katoh Y, Oyake T, Hayashi N, Satoh K, Hatayama I, et al. 1997. An Nrf2/ small Maf heterodimer mediates the induction of phase II detoxifying enzyme genes through antioxidant response elements. Biochem Biophys Res Commun 236: 313-322.

King-Jones K, Horner M, Lam G, Thummel C. 2006. The DHR96 nuclear receptor regulates xenobiotic responses in Drosophila. Cell Metab 4: 37-48.

Li X, Schuler MA, Berenbaum MR. 2007. Molecular mechanisms of metabolic resistance to synthetic and natural xenobiotics. Annu Rev Entomol 52: 231-253.

Maglich JM, Stoltz CM, Goodwin B, Hawkins-Brown D, Moore JT, Kliewer SA. 2002. Nuclear pregnane $\times$ receptor and constitutive androstane receptor regulate overlapping but distinct sets of genes involved in xenobiotic detoxification. Mol Pharmacol 62: 638-646.

Maitra S, Dombrowski SM, Waters LC, Ganguly R. 1996. Three second chromosome-linked clustered Cyp6 genes show differential constitutive and barbital-induced expression in DDT-resistant and susceptible strains of Drosophila melanogaster. Gene 180: 165-171.

Maitra S, Dombrowski SM, Basu M, Raustol O, Waters LC, Ganguly R. 2000. Factors on the third chromosome affect the level of cyp6a2 and cyp6a8 expression in Drosophila melanogaster. Gene 248: 147-156.

McDonnell CM, Brown RP, Berenbaum MR, Schuler MA. 2004. Conserved regulatory elements in the promoters of two allelochemical-inducible cytochrome P450 genes differentially regulate transcription. Insect Biochem Mol Biol 34: 1129-1139.

McGinnis N, Ragnhildstveit E, Veraksa A, McGinnis W. 1998. A cap ' $\mathrm{n}$ ' collar protein isoform contains a selective Hox repressor function. Development 125: 4553-4564.

McMillan B, Bradfield CA. 2007. The aryl hydrocarbon receptor sans xenobiotics: endogenous function in genetic model systems. Mol Pharmacol 72: 487-498.

Morra R, Kuruganti S, Lam V, Lucchesi JC, Ganguly R. 2010. Functional analysis of the cis-acting elements responsible for the induction of the Cyp6a8 and Cyp6g1 genes of Drosophila melanogaster by DDT, phenobarbital and caffeine. Insect Mol Biol 19: 121-130.

Morrison PD, Murray RM. 2005. Schizophrenia. Curr Biol 15: R980-R984. doi: 10.1016/j.cub.2005.11.059.

Negre N, Brown CD, Ma L, Bristow CA, Miller SW, Wagner U, Kheradpour P, Eaton ML, Loriaux P, Sealfon R, et al. 2011. A cis-regulatory map of the Drosophila genome. Nature 471: 527-531.

Nguyen T, Nioi P, Pickett CB. 2009. The Nrf2-antioxidant response element signaling pathway and its activation by oxidative stress. J Biol Chem 284: 13291-13295.

Pascussi JM, Gerbal-Chaloin S, Duret C, Daujat-Chavanieu M, Vilarem MJ, Maurel P. 2008. The tangle of nuclear receptors that controls xenobiotic metabolism and transport: crosstalk and consequences. Annu Rev Pharmacol Toxicol 48: $1-32$.

Perry T, Batterham P, Daborn PJ. 2011. The biology of insecticidal activity and resistance. Insect Biochem Mol Biol 41: 411-422.

Radyuk SN, Michalak K, Klichko VI, Benes J, Orr WC. 2010. Peroxiredoxin 5 modulates immune response in Drosophila. Biochim Biophys Acta 1800: 1153-1163.

Rowlands JC, Gustafsson JA. 1997. Aryl hydrocarbon receptormediated signal transduction. Crit Rev Toxicol 27: 109134.

Sackton TB, Clark AG. 2009. Comparative profiling of the transcriptional response to infection in two species of Drosophila by short-read cDNA sequencing. BMC Genomics 10: 259.

Schecter A, Birnbaum L, Ryan JJ, Constable JD. 2006. Dioxins: an overview. Environ Res 101: 419-428.

Sun W, Margam VM, Sun L, Buczkowski G, Bennett GW, Schemerhorn B, Muir WM, Pittendrigh BR. 2006. Genomewide analysis of phenobarbital-inducible genes in Drosophila melanogaster. Insect Mol Biol 15: 455-464.

Sykiotis GP, Bohmann D. 2008. Keap1/Nrf2 signaling regulates oxidative stress tolerance and lifespan in Drosophila. Dev Cell 14: 76-85.

Sykiotis GP, Bohmann D. 2010. Stress-activated cap'n'collar transcription factors in aging and human disease. Sci Signal 3: re3. doi: 10.1126/scisignal.3112re3.

Szeri F, Ilias A, Pomozi V, Robinow S, Bakos E, Varadi A. 2009. The high turnover Drosophila multidrug resistance-associated protein shares the biochemical features of its human orthologues. Biochim Biophys Acta 1788: 402-409.

Veraksa A, McGinnis N, Li X, Mohler J, McGinnis W. 2000. Cap ' $n$ ' collar B cooperates with a small Maf subunit to specify pharyngeal development and suppress deformed homeotic function in the Drosophila head. Development 127: 4023 4037.

Vontas J, Blass C, Koutsos AC, David JP, Kafatos FC, Louis C, Hemingway J, Christophides GK, Ranson H. 2005. Gene expression in insecticide resistant and susceptible Anopheles gambiae strains constitutively or after insecticide exposure. Insect Mol Biol 14: 509-521. 
Misra et al.

Wei P, Zhang J, Dowhan DH, Han Y, Moore DD. 2002. Specific and overlapping functions of the nuclear hormone receptors CAR and PXR in xenobiotic response. Pharmacogenomics I 2: 117-126.

Willoughby L, Chung H, Lumb C, Robin C, Batterham P, Daborn PJ. 2006. A comparison of Drosophila melanogaster detoxification gene induction responses for six insecticides, caffeine and phenobarbital. Insect Biochem Mol Biol 36: 934942.

Wilson TG, Fabian J. 1986. A Drosophila melanogaster mutant resistant to a chemical analog of juvenile hormone. Dev Biol 118: 190-201.

Xu C, Li CY, Kong AN. 2005. Induction of phase I, II and III drug metabolism/transport by xenobiotics. Arch Pharm Res 28: 249-268. 


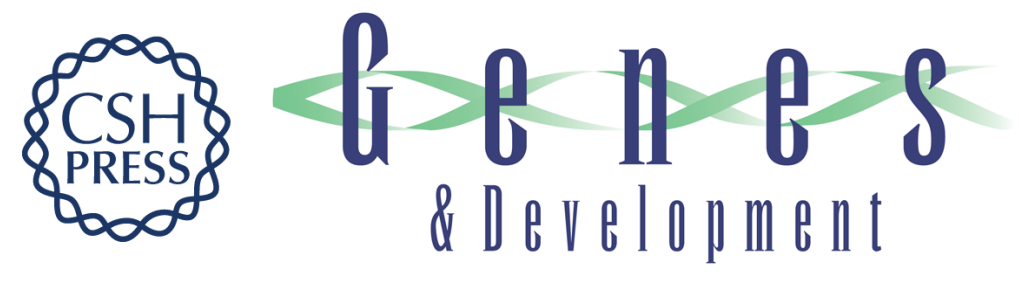

\section{Transcriptional regulation of xenobiotic detoxification in Drosophila}

Jyoti R. Misra, Michael A. Horner, Geanette Lam, et al.

Genes Dev. 2011, 25:

Access the most recent version at doi:10.1101/gad.17280911

Supplemental

Material

References

This article cites 50 articles, 14 of which can be accessed free at:

http://genesdev.cshlp.org/content/25/17/1796.full.html\#ref-list-1

\section{License}

Email Alerting Service

http://genesdev.cshlp.org/content/suppl/2011/09/07/25.17.1796.DC1 right corner of the article or click here.

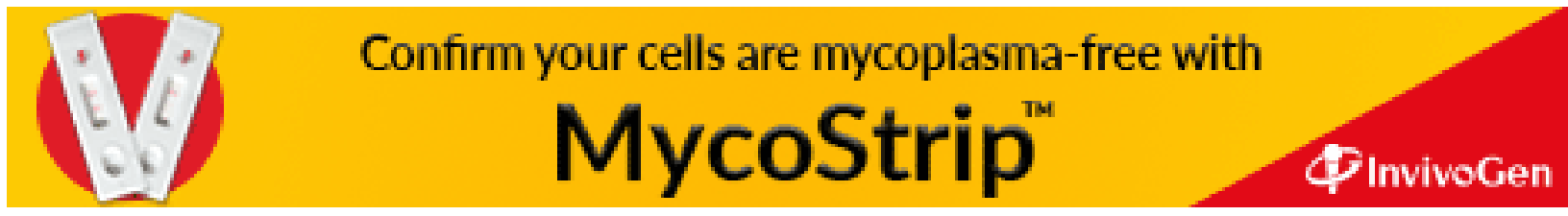

